\title{
Unilateral thalamic and pallidal deep brain stimulation for idiopathic hemidystonia: results of individual and combined stimulations. Case report
}

\author{
Victor Goulenko, MD, Paulo Luiz da Costa Cruz, MD, and Paulo Niemeyer Filho, MD, PhD \\ Department of Neurosurgery, Paulo Niemeyer State Brain Institute, Rio de Janeiro, Brazil
}

Pallidal stimulation has been the usual surgical treatment for dystonia in the last decades. The continuous investigation of the physiopathology and the motor pathways involved leads to the search for complementary targets to improve results. The authors present the case of a 37-year-old woman who had suffered from idiopathic hemidystonia with hyperkinetic and hypokinetic movements for 11 years, and who was treated with deep brain stimulation. A brief literature review is also provided. The globus pallidus internus and the ventral intermediate/ventral oral posterior complex of the thalamus were stimulated separately and simultaneously for 3 months and compared using the Burke-Fahn-Marsden Dystonia Rating Scale and the Global Dystonia Severity Rating Scale, with a 3.5-year follow-up. The synergism of multiple-target stimulation resulted in a complete improvement of the mixed dystonic symptoms.

https://thejns.org/doi/abs/10.3171/2017.4.FOCUS17134

KEY WORDS deep brain stimulation; hemidystonia; pallidal; thalamic; simultaneous

A FTER the occurrence of brain damage with motor deficits, neuroplasticity leads to the development of an aberrant motor network leading to dystonic movements on a single side of the body, characterizing hemidystonia. When the cause of the brain insult is not found in the clinical investigation, it is termed idiopathic hemidystonia. Pharmacotherapy is the first-line treatment, associated with multidisciplinary therapy. Stereotactic ablation of the globus pallidus internus (GPi) and thalamus are being massively replaced by deep brain stimulation (DBS). ${ }^{5,9,10,14,21,22,24}$

Pallidal stimulation is indicated for several types of dystonia, especially the primary type with hyperkinetic movements. However, other targets such as the thalamus and the subthalamic nucleus (STN) show good results in tremor, myoclonus, and task-specific dystonia, and for rescue therapy. ${ }^{4-6,8-10,12-14,16-18,20}$ A precise target has not been established for each type of dystonia, ${ }^{14,16}$ and it is known that the etiology interferes with the surgical outcome.?

Hemidystonia is not well treated by a single target be- cause of its complex rearrangement of the motor network, ${ }^{4}$ and hypokinetic symptoms don't respond well to pallidal modulation. ${ }^{4,14}$ Complementary targets have been used

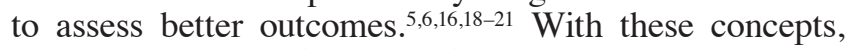
multiple-target DBS was performed to treat the phasic and nonphasic symptoms in a hemidystonic patient. Each target was stimulated individually and then combined for 3 -month periods, and their results were then compared.

\section{Case Report}

History

This 37-year-old right-handed woman began experiencing an involuntary muscular contraction at the left inferior limb 11 years earlier, which soon progressed to the superior limb and face. There was no history of trauma or previous motor disturbance and no family history of movement disorders. Brain and cervical spine MRI sequences showed no evidence of injuries or acute embolic lesions. Electroneuromyography suggested dystonic activities, ex-

ABBREVIATIONS BFMDRS = Burke-Fahn-Marsden Dystonia Rating Scale; DBS = deep brain stimulation; GDSRS = Global Dystonia Severity Rating Scale; GPi = globus pallidus internus; IPG = implantable pulse generator; STN = subthalamic nucleus; Vim = ventral intermediate; Vo = ventral oral; Vop = Vo posterior. 

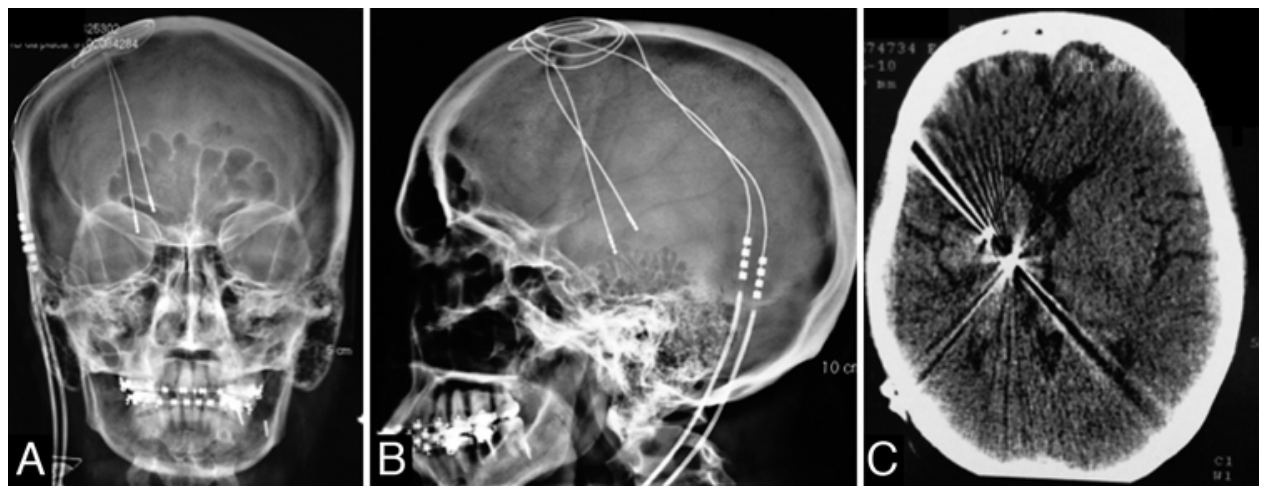

FIG. 1. Coronal (A) and sagittal (B) radiographs and axial CT scan (C) showing a single bur hole entrance and final electrode positioning.

cluding peripheral disturbances. Genetic testing wasn't performed. The patient began pharmacological therapy and botulinum toxin, with minimum effect. Worsening symptoms led to a continuous loss in quality of life, severe pain, and inability to stand, with the patient requiring a wheel chair.

\section{Examination}

The patient presented abnormal movements only on her left side, with moderate hyperkinetic (phasic) arm movements accentuated by delicate tasks, resulting in functional limitation in most activities. The leg was hypokinetic (nonphasic or tonic), which led to a permanently extended foot and the inability to flex her knee. She also had occasional blinking and grimacing. The Burke-Fahn-Marsden Dystonia Rating Scale (BFMDRS) score ${ }^{2}$ was 26 and the Global Dystonia Severity Rating Scale (GDSRS) score ${ }^{3}$ was 27.

\section{Operative Technique}

The head frame was attached to the skull under local anesthesia, followed by a high-resolution stereotactic CT scan. The images were fused with preoperative 1.5-T MRI sequences. After determining the anterior and posterior commissures, the posteroventrolateral part of the GPi and the ventral intermediate (Vim)/ventral oral posterior (Vop) nucleus complex was localized by direct and indirect targeting techniques.

The patient was then sedated with Precedex and midazolam, remaining awake throughout the procedure. Using local anesthetic, an L-shaped frontal incision and a single 14-mm bur hole at the entry point were performed. Microelectrode mapping showed greater motor cell activities in the leg compared with the arm on both targets, and macrostimulation had good motor responses at arm/hand and leg/foot testing. Surgical glue helped prevent CSF loss, minimizing brain shift. Medtronic model 3389 leads were implanted at the GPi and Vim/Vop. A rechargeable implantable pulse generator (IPG) was placed in the subclavicular area under general anesthesia on the same day.

\section{Postoperative Course}

Radiography confirmed correct system connection and showed both leads entering through a single bur hole. The
CT scans were merged to the stereotactic planning images, confirming adequate final position of the leads. Images are shown in Fig. 1. The patient was discharged after 24 hours of observation in the intensive care unit without any complications. The IPG was kept turned off.

At the first evaluation 1 week after surgery, the patient had better voluntary foot movement; she was able to stand and walk a short distance by herself, but needed assistance most of the time. There was a complete improvement of the facial dystonia but no significant changes in the arm. This initial response was attributed to the implantation effect. The BFMDRS score was 18 and the GDSRS score was 20 (Video 1).

VIDEO 1. Clip showing the patient 1 week after surgery with stimulation off, with implantation effect. Copyright Victor Goulenko. Published with permission. Click here to view.

Stimulation of the GPi was started, and the patient had an immediate subtle knee flexion (Video 2).

VIDEO 2. Clip showing the patient 1 week after surgery, with immediate effect of pallidal stimulation. Copyright Victor Goulenko. Published with permission. Click here to view.

After 3 months of pallidal stimulation, the patient's gait improved due to better leg movements, but she still needed assistance with longer walks. The patient reported that the arm was slightly ameliorated, but without functional changes. Her BFMDRS score was 12 and her GDSRS score was 7. The GPi stimulation was turned off to start the thalamic test, which had the same benefits and the same score as the BFMDRS and GDSRS after 3 months. The only difference was the involvement of other muscle groups in the leg.

With the simultaneous test the arm dystonia disappeared, with complete functional movements. There was a slight dystonic gait without significant loss of independence. These results were obtained with $120 \mu$ sec, 130 $\mathrm{Hz}$, and $5 \mathrm{~V}$ stimulation in the GPi (bipolar, 2+, and 1-), and $2.5 \mathrm{~V}$ in the Vim/Vop (monopolar, case+, 2-, 1-). The BFMDRS and the GDSRS had drastic reductions, both scoring 1.

Because the combined stimulation had greater response, continuous programming of both targets aimed to improve leg movements. In the last year, the stimulation parameters were kept unchanged. Turning off the stimulation led to worsened speech and gait, with a compensatory 
trunk inclination, slight grimacing, and intense contractions in the affected hand limiting the evaluation, with BFMDRS and GDSRS scores of 25.5 and 19, respectively. With a low battery charge, the patient only presents with worsening speech and gait, with a BFMDRS score of 3 and a GDSRS score of 2; but when the battery is fully charged, both scores are 0 . The final programming was as follows: GPi in bipolar $(2+, 1-)$ with $6.5 \mathrm{~V}, 170 \mu \mathrm{sec}$, and 185 Hz stimulation; Vim/Vop in monopolar (case+, 2-, 1-) with $3.5 \mathrm{~V}, 160 \mathrm{usec}$, and $185 \mathrm{~Hz}$ stimulation.

There was no surgical complication and no evidence of infection or system malfunction during the follow-up. After the surgery, the patient had weight gain that caused difficulty in recharging the IPG battery, necessitating superficialization of the IPG. During the thalamic test she underwent bariatric surgery, with the stimulation turned off only during the procedure.

Undesirable stimulation effects occurred when GPi programming was changed from bipolar $(2+, 1-)$ to monopolar (case+, 2-, 1-), manifested by speech difficulty and superior limb contractions. Progressively increasing the pulse width up to $160 \mu \mathrm{sec}$ and $170 \mu \mathrm{sec}$ was tolerated, but 180 usec caused gait disturbance in both targets. At 3.5 years after the surgery, the patient didn't present any dystonic movements and had a normal gait. She was able to graduate as a paramedic, without any physical limitations (Video 3).

VIDEO 3. Clip showing the patient 3.5 years after surgery, with total functional movements (first part); and at 3.7 years after surgery, with GPi and Vim off and with dystonia (second part). Copyright Victor Goulenko. Published with permission. Click here to view.

\section{Discussion}

Hemidystonia can be related to a disturbance in the cortico-striato-pallido-thalamo-cortical and cerebellothalamo-cortical pathways, and also to alteration in cortical activities after maladaptive neuroplasticity.,11,21 The different topography of brain injury influences a variety of new, aberrant networks, explaining the heterogenic presentation in dystonia. ${ }^{4}$

Widespread bilateral cortical overactivity with interhemispheric transfer is related to hypokinetic or a mixedpresentation dystonia. ${ }^{4}$ An oscillatory activation (10-30 $\mathrm{Hz}$ ) in pallidal-thalamic relay ${ }^{7}$ with ipsilateral cerebellar and contralateral sensorimotor cortex recruitment are also related. ${ }^{4}$ These manifestations are due to damage in the pallidal and postpallidal output pathways. ${ }^{4}$ Hyperkinetic patients have decreased activity in contralateral thalamus, GPi, and cortex (cortico-striato-pallido-thalamic circuit), with preserved postpallidal structures. ${ }^{4}$

The use of GPi-DBS has been extensively studied and described thoroughly in the literature. It modulates pallidal bursts to the thalamocortical relay, ${ }^{7,15}$ but it is compromised by the abnormal plasticity in dystonia. ${ }^{6,7}$ Despite the reference of Vop connection to the cerebellum, 5,17,21 studies with its direct reading, ${ }^{11,15}$ including an analysis of pallidal stimulation effects on Vop, showed that "Vop is a major target of GPi efferents" $6,11,15,16$ and attributed to Vim the cerebellar connection. ${ }^{11,15,16}$ In another study analyzing the pallidal-thalamic circuit, after GPi and Vim DBS for hemidystonia, a 5- to $18-\mathrm{Hz}$ activity was observed, but some of the readings recorded at the Vim could have originated from the adjacent pallidal-receiving thalamus (the Vop). ${ }^{23}$ A multitarget neuromodulation therapy for greater clinical results is justified by acting on both disturbed pathways, ${ }^{1,4,6,21}$ as was observed in the presented case.

Lenz et al. ${ }^{11}$ studied the electromyographic response after Vim and Vop microstimulation, finding altered spontaneous activity in the Vop and somatic sensory reorganization in the Vim with dystonic patients. Stimulating the Vim in the dystonic group produced muscle activities through these modified sensory maps, whereas in the nondystonic group motor alterations were not produced. ${ }^{11}$

In this manner, hyperkinetic presentation is related to GPi and Vop in the cortico-striato-pallido-thalamocortical pathway, and hypokinesia with Vim through the cerebello-thalamo-cortical pathway. It helps to explain the lack of response to GPi DBS in hypokinetic patients, ${ }^{4,14}$ and in those with interhemispheric overactive areas on functional MRI (also related to tonic dystonia). ${ }^{4}$

There are few studies regarding pallidal and thalamic stimulation, but they have heterogeneous results. $5,6,8,9,16-19$, 21,22,24 Motor improvement with GPi DBS, Vim DBS, or with both simultaneously, might be explained by the different types of dystonia evaluated and by targeting. ${ }^{22,24}$ Not all studies were designed initially for combined stimulation; in some studies a secondary surgery was performed to implant electrodes at different targets after failure of the first. ${ }^{9,17,24}$ A 2006 meta-analysis ${ }^{9}$ showed that the GPi stimulation had significantly better outcomes compared with the posterior portion of the ventrolateral nucleus of the thalamus, which includes Vim and part of the ventral oral (Vo) complex, 9,16 but the majority of the patients reviewed had primary dystonia. ${ }^{9}$ The thalamic anatomy has many descriptions that could lead to targeting changes, and the fact that it has unclear and overlapped nuclear borders interferes with the clinical analysis. Even changes inside a thalamic nucleus can have a different effect; for example, the posteromedial part of the Vim can treat tremor and drug-induced dyskinesias, and the anterolateral part can only treat tremor, ${ }^{22}$ which can also be explained by stimulation of a nearby nucleus.

Combined stimulation had a better outcome than individual GPi or Vim DBS in other studies, 6,18,19 having prolonged motor improvement for up to 10 years. This method was also safe, with greater quality of life when evaluated according to the general perception of health and change of health scores. ${ }^{6}$ The possibility of covering all the clinical presentations is a direct benefit of multiple targets. It also allowed the reduction of stimulation-related adverse events - e.g., undesirable effects of Vim stimulation could be ameliorated with the combination of the GPi by reducing the necessity for increased amplitude. ${ }^{6}$

Slotty et al. ${ }^{21}$ presented the case of a hemidystonia that did not benefit from individual stimulation, but when the methods were combined, it led to a decrease of $28 \%$ in the BFMDRS score. The follow-up of only 6 months might not represent the benefits in their entirety. The final programming was in the GPi: $120 \mu \mathrm{sec}, 185 \mathrm{~Hz}, 4.5 \mathrm{~V}, 1+, 0-$; and in the Vim/Vop: $60 \mu \mathrm{sec}, 185 \mathrm{~Hz}, 4.0 \mathrm{~V}, 0+, 4-$.

Oropilla et al. ${ }^{19}$ observed that myoclonic symptoms im- 
proved with the GPi DBS, but the tremor only responded when the Vim DBS was activated (although these authors considered that they actually stimulated the Vop), with $80 \%$ of improvement after 2-year follow-up. The final programming was in the GPi: $120 \mu \mathrm{sec}, 140 \mathrm{~Hz}, 2.3 \mathrm{~V}$, case+, 2-; and in the Vim: $90 \mu \mathrm{sec}, 140 \mathrm{~Hz}, 2.1 \mathrm{~V}$, case+, 2-.

A retrospective study of GPi and Vim DBS for myoclonic dystonia in 10 patients reported that the outcome was slightly superior with combined stimulation. The final programming settings were not mentioned. ${ }^{6}$

Focal hand dystonia associated with contralateral writer's cramp was treated with unilateral GPi and Vo DBS. The improvement happened only with combined stimulation; there was no change when stimulation was individualized. Final programming was in the GPi: $450 \mu \mathrm{sec}, 130$ $\mathrm{Hz}, 1.0 \mathrm{~V}$; and in the ventral oral complex: $60 \mu \mathrm{sec}, 130$ $\mathrm{Hz}, 3.0 \mathrm{~V} .^{5}$

One case of dystonia with tremor had a $45 \%$ decrease in symptoms in a 4-year follow-up of bilateral GPi and Vim DBS. The final programming was in the left GPi: 120 $\mu$ sec, $155 \mathrm{~Hz}, 3.2 \mathrm{~V}$, case+, 3-; and right GPi: $180 \mu \mathrm{sec}, 60$ $\mathrm{Hz}, 2.5 \mathrm{~V}$, case+, 2-, 3-; left Vim: $120 \mu \mathrm{sec}, 155 \mathrm{~Hz}, 3.1 \mathrm{~V}$, 3+, 2-; and right Vim: 180 usec, $60 \mathrm{~Hz}, 2.3 \mathrm{~V}$, case+, 2-. ${ }^{16}$

Two cases of neuroacanthocytosis had the GPi and Vo complex as targets bilaterally, as reported by Nakano et al. The first one had $40 \%$ improvement and the second had $48 \%$. The first patient's final programming was in the GPi: $90 \mu \mathrm{sec}, 160 \mathrm{~Hz}, 3.5 \mathrm{~V}$, case+, 0-, 1-; and in the Vo complex: $60 \mu \mathrm{sec}, 160 \mathrm{~Hz}, 3.0 \mathrm{~V}, 3+, 2-$. In the second patient the GPi programming was: $90 \mu \mathrm{sec}, 160 \mathrm{~Hz}, 3.5 \mathrm{~V}$, case+, 0-, 1-; and the Vo complex was: $60 \mu \mathrm{sec}, 160 \mathrm{~Hz}$, $3.0 \mathrm{~V}, 3+, 2-.^{18}$

Other studies did not find benefits with thalamic stimulation. ${ }^{17,22,24}$ In a retrospective evaluation of 19 patients, Vercueil et al. ${ }^{24}$ found that those treated with GPi DBS had a better outcome, and 3 patients in the thalamic group had rescue GPi DBS, with subsequent improvement. One hemidystonic patient lost thalamic stimulation benefits; this individual had a greater response with pallidal stimulation. ${ }^{24}$ In a case of tardive dystonia, Trottenberg et al. reported that the Vim did not influence the hyperkinetic movements. ${ }^{22}$

The variety of etiologies, the physiopathology, the topography of the brain insult, and the different ways that a compensatory motor network is formed explains the heterogeneity of outcomes and different programming settings. A good evaluation of each patient will influence which target is chosen. Stimulating a single nucleus might not completely control all of the symptoms, necessitating an additional target.

The GPi would influence the phasic component, affecting the pallido-thalamo-cortical circuit in our patient, associated with modulation of the Vop. However, it would not influence the nonphasic component. Another target was needed for complementation. The Vim modulates the cerebello-thalamo-cortical circuit affecting the hypokinetic movement, but not the hyperkinetic movement. Theoretically, the accentuated arm movements would be better treated by stimulation of the GPi and Vop, and the tonic leg by Vim/Vop. We observed that a clinical response happened with both individual stimulations, but the synergism led to an optimized response, with complete improvement of these opposed types of movements.

A sustained and prolonged effect on symptoms after stimulation cessation has been described ${ }^{6,19,24}$ and has been attributed to changes in neuroplasticity. ${ }^{6,7}$ The same results that occurred in the present case, when GPi and Vim were stimulated individually, might be related to a delayed pallidal or immediate Vim stimulation effect, but we consider that their combined stimulation is the main reason, because different muscle groups were affected by each target. This sustained effect also explains the persistent good motor response when the battery charge is low, and the speech disturbance was attributed to the progressiveness of dystonia. Dysarthria happened when a different configuration in the GPi was tested, as observed by Mure et al..$^{17}$ in a patient with DYT6 primary dystonia, although Gruber et al. ${ }^{6}$ only noticed this adverse event in Vim DBS.

Other clinical manifestations were pain relief that had improvement associated with DBS in primary and secondary dystonia (especially in hypokinesias after better joint movement ${ }^{14}$ ), and weight gain that wasn't attributed to either the GPi or the Vim/Vop stimulation; it was only statistically associated with STN DBS. ${ }^{13}$

\section{Conclusions}

Heterogeneous results of GPi and Vim/Vop stimulation have been reported, but none of the cases had such a dramatic clinical response with simultaneous stimulation as in the case reported here. Because of the mixed presentation, a single-target treatment would not completely benefit the patient. The phasic presentation would be well treated by the GPi DBS, but not the nonphasic symptom. Therefore, stimulating the Vim/Vop would better complement the clinical response. The main life-limiting complaint was leg dystonia, and during programming it was evident that each target interfered in different muscle groups, producing better results. The synergism in stimulating both of those pathways led to an optimized result, promoting improved quality of life, with total control of the hypo- and hyperkinetic dystonia.

\section{References}

1. Álvarez M, Quintanal N, Díaz A, Prince J, García I, Carballo $\mathrm{M}$, et al: Dystonia and tremor secondary to thalamic infarction successfully treated with thalamotomy of the ventralis intermedius nucleus. Mov Disord 29:1188-1190, 2014

2. Burke RE, Fahn S, Marsden CD, Bressman SB, Moskowitz $\mathrm{C}$, Friedman J: Validity and reliability of a rating scale for the primary torsion dystonias. Neurology 35:73-77, 1985

3. Comella CL, Leurgans S, Wuu J, Stebbins GT, Chmura T: Rating scales for dystonia: a multicenter assessment. Mov Disord 18:303-312, 2003

4. Gonzalez V, Le Bars E, Cif L, van Dokkum LEH, Laffont I, Bonafé A, et al: The reorganization of motor network in hemidystonia from the perspective of deep brain stimulation. Brain Imaging Behav 9:223-235, 2015

5. Goto S, Shimazu H, Matsuzaki K, Tamura T, Murase N, Nagahiro S, et al: Thalamic Vo-complex vs pallidal deep brain stimulation for focal hand dystonia. Neurology 70:1500-1501, 2008

6. Gruber D, Kühn AA, Schoenecker T, Kivi A, Trottenberg T, 
Hoffmann KT, et al: Pallidal and thalamic deep brain stimulation in myoclonus-dystonia. Mov Disord 25:1733-1743, 2010

7. Guo Y, Park C, Worth RM, Rubchinsky LL: Basal ganglia modulation of thalamocortical relay in Parkinson's disease and dystonia. Front Comput Neurosci 7:124, 2013

8. Guzzi G, Della Torre A, Chirchiglia D, Volpentesta G, Lavano A: Critical reappraisal of DBS targeting for movement disorders. J Neurosurg Sci 60:181-188, 2016

9. Holloway KL, Baron MS, Brown R, Cifu DX, Carne W, Ramakrishnan V: Deep brain stimulation for dystonia: a metaanalysis. Neuromodulation 9:253-261, 2006

10. Kim JP, Chang WS, Park YS, Chang JW: Bilateral globus pallidus internus deep brain stimulation for DYT1+ generalized dystonia with previously received bilateral thalamotomy and unilateral pallidotomy. Stereotact Funct Neurosurg 89:205-209, 2011

11. Lenz FA, Jaeger CJ, Seike MS, Lin YC, Reich SG, DeLong MR, et al: Thalamic single neuron activity in patients with dystonia: dystonia-related activity and somatic sensory reorganization. J Neurophysiol 82:2372-2392, 1999

12. Matias CM, Silva D, Machado AG, Cooper SE: "Rescue" of bilateral subthalamic stimulation by bilateral pallidal stimulation: case report. J Neurosurg 124:417-421, 2016

13. Mills KA, Scherzer R, Starr PA, Ostrem JL: Weight change after globus pallidus internus or subthalamic nucleus deep brain stimulation in Parkinson's disease and dystonia. Stereotact Funct Neurosurg 90:386-393, 2012

14. Mills KA, Starr PA, Ostrem JL: Neuromodulation for dystonia: target and patient selection. Neurosurg Clin $\mathbf{N}$ Am 25:59-75, 2014

15. Montgomery EB Jr: Effects of GPi stimulation on human thalamic neuronal activity. Clin Neurophysiol 117:26912702,2006

16. Morishita T, Foote KD, Haq IU, Zeilman P, Jacobson CE, Okun MS: Should we consider Vim thalamic deep brain stimulation for select cases of severe refractory dystonic tremor. Stereotact Funct Neurosurg 88:98-104, 2010

17. Mure H, Morigaki R, Koizumi H, Okita S, Kawarai T, Miyamoto R, et al: Deep brain stimulation of the thalamic ventral lateral anterior nucleus for DYT6 dystonia. Stereotact Funct Neurosurg 92:393-396, 2014

18. Nakano N, Miyauchi M, Nakanishi K, Saigoh K, Mitsui Y, Kato A: Successful combination of pallidal and thalamic stimulation for intractable involuntary movements in patients with neuroacanthocytosis. World Neurosurg 84:1177. e1-1177.e7, 2015

19. Oropilla JQL, Diesta CCE, Itthimathin P, Suchowersky O, Kiss ZHT: Both thalamic and pallidal deep brain stimulation for myoclonic dystonia. J Neurosurg 112:1267-1270, 2010

20. Schjerling L, Hjermind LE, Jespersen B, Madsen FF, Brennum J, Jensen SR, et al: A randomized double-blind crossover trial comparing subthalamic and pallidal deep brain stimulation for dystonia. J Neurosurg 119:1537-1545, 2013

21. Slotty PJ, Poologaindran A, Honey CR: A prospective, randomized, blinded assessment of multitarget thalamic and pallidal deep brain stimulation in a case of hemidystonia. Clin Neurol Neurosurg 138:16-19, 2015

22. Trottenberg T, Paul G, Meissner W, Maier-Hauff K, Taschner C, Kupsch A: Pallidal and thalamic neurostimulation in severe tardive dystonia. J Neurol Neurosurg Psychiatry 70:557-559, 2001

23. Tsang EW, Hamani C, Moro E, Mazzella F, Lozano AM, Yeh IJ, et al: Prominent 5-18 Hz oscillations in the pallidal-thalamic circuit in secondary dystonia. Neurology 78:361-363, 2012

24. Vercueil L, Pollak P, Fraix V, Caputo E, Moro E, Benazzouz A, et al: Deep brain stimulation in the treatment of severe dystonia. J Neurol 248:695-700, 2001

\section{Disclosures}

The authors report no conflict of interest concerning the materials or methods used in this study or the findings specified in this paper.

\section{Author Contributions}

Conception and design: Goulenko, Cruz. Acquisition of data: Goulenko. Analysis and interpretation of data: Goulenko. Drafting the article: Goulenko. Critically revising the article: all authors. Reviewed submitted version of manuscript: Goulenko, Cruz. Approved the final version of the manuscript on behalf of all authors: Goulenko. Study supervision: Cruz, Niemeyer Filho.

\section{Supplemental Information \\ Videos}

Video 1. https://vimeo.com/216834348.

Video 2 . https://vimeo.com/216834452.

Video 3. https://vimeo.com/216834584.

\section{Correspondence}

Victor Goulenko, Departamento de Neurocirurgia, Instituto Estadual do Cérebro Paulo Niemeyer, Rua do Rezende, 156, Rio de Janeiro, RJ 20231-092, Brazil. email: vgoulenko@gmail.com. 